\title{
RARE CASE REPORT OF ACUTE MYOCARDIAL INFARCTION AS AN INITIAL PRESENTATION OF THYROTOXICOCIS
}

Surbhi Chaturvedi1 ${ }^{1}$, Niharika Sharma ${ }^{2}$, Ankit Manglunia3 ${ }^{3}$, Prakhar Garg ${ }^{4}$, Ganesh Narain Saxena ${ }^{5}$

\section{HOW TO CITE THIS ARTICLE:}

Surbhi Chaturvedi, Niharika Sharma, Ankit Manglunia, Prakhar Garg, Ganesh Narain Saxena. "Rare Case Report of Acute Myocardial Infarction as an Initial Presentation of Thyrotoxicocis". Journal of Evolution of Medical and Dental Sciences 2015; Vol. 4, Issue 54, July 06; Page: 9515-9517, DOI: 10.14260/jemds/2015/1377

ABSTRACT: Thyrotoxicosis is associated with increased cardiovascular morbidity and mortality, primarily due to heart failure and thromboembolism. However, the relationship between thyroid hormone excess and the cardiac complications of angina pectoris and myocardial infarction remains largely speculative. Moreover, few studies have been reported on the effect of thyroid hormone levels within normal range on coronary artery disease (CAD). Thyroid hormones may directly influence myocardial oxygen supply and demand and cause a critical imbalance resulting in angina pectoris and myocardial infarction ${ }^{1}$. We present the case where acute MI was an initial presentation of thyrotoxicosis in a young man of 30 years of age who had been entirely fit \& well prior to this acute coronary event.

CASE REPORT: A 30 year old male patient presented with sudden onset sub-sternal, squeezing nature chest pain radiating to the left arm with palpitations, sweating and tremors for last 2 hours. No associated history of exertion, fever, coughs, dyspnoea or heat intolerance. No history of diabetes, hypertension, dyslipidaemia or any other chronic disorder. Family history was insignificant. He did not use drugs or alcohol, and except for a history of smoking cigarette quarter of pack per year, he had healthy attributes.

On Physical examination the blood pressure-130/80 mmHg, heart rate-100bpm, BMI-24.6 with normal jugular venous pressure and carotid pulse. The head and neck examination were normal, no exophthalmos. Cardiac auscultation was within normal limits except for the tachycardia. No murmurs heard. Other systemic examination was within normal limits.

Urgent ECG done which revealed ST segment elevation with $\mathrm{T}$ wave inversion in anterior leads. Patient was loaded with aspirin, clopidogrel and atorvastatin as an urgent measure and admitted.

Laboratory investigations revealed normal electrolytes and kidney function test. Complete blood count was normal with slightly deranged LFT [S. bilirubin T-2.2, D-1.3, SGOT-188, SGPT-71, ALP 173] and Plasma Lipids (Total cholesterol 127mg/dl, HDL 36mg/dl, LDL 77.2mg/dl, VLDL $13.8 \mathrm{mg} / \mathrm{dl}$, Triglycerides $69 \mathrm{mg} / \mathrm{dl}$ ). Random blood sugar was $212 \mathrm{mg} / \mathrm{dl}$. CK-MB was $30.2 \mathrm{ng} / \mathrm{ml}$ (Normal: 0.6-6.4ng/ml).2D echo shows LVEF 45\% with mild LV dysfunction. Emergency coronary angiography was performed. This revealed a massive spasm of left anterior descending (LAD) coronary artery. There was critical narrowing of the LAD (70\%) in apical segment with possible superimposed thrombus. Primary PTCA was advised. Recanalization LAD was successfully done. He was started on standard medication used in patients after myocardial infarction (Aspirin, clopidogril, acitrom, cardarone, lvabradin, rosuvastatin). Post PTCA echocardiography was done which showed regional wall motion abnormality with mild impairment of LV function and mild MR. He continued to have tachycardia and tremors. Thyroid function test was advised. It revealed free-T4 7.8 (Normal: 3.1-6.8pmol/l), free-T3 34.96 (Normal: 12.0-22.0pmol/l) and TSH 0.034 (Normal: 0.274.2IU/ml), 


\section{CASE REPORT}

Thyroglobulin 2.30ng/mL (Normal: 1.4-78.0ng/mL) and Thyroglobulin antibody 10.11IU/mL (Normal: $<115.0 \mathrm{IU} / \mathrm{mL}$ ) which was suggestive of thyrotoxicosis. Tremors and tachycardia also improved after he was started on anti-thyrox medication and is doing well on follow up.

DISCUSSION: Acute Myocardial Infarction is a condition in which there is an inadequate supply of blood and oxygen to a portion of the myocardium; it typically occurs when there is an imbalance between myocardial oxygen supply and demand. ${ }^{1}$

Hyperthyroidism is associated with many heart diseases. Thyrotoxic state has a relationship with coronary spasm. Thyroid hormones affect cardiovascular system by increasing stroke volume and heart rate. ${ }^{2}$ Excessive thyroid hormones are linked to many heart diseases such as angina, myocardial infarction, arrhythmia and sudden death. ${ }^{3}$ The possible mechanisms of coronary occlusion with thyrotoxicosis include: significant underlying coronary atherosclerosis, direct damage to coronary artery and coronary embolization. ${ }^{4}$ Several hypothesis have been proposed for the mechanism of thyroid hormone and coronary artery spasm. The basic idea is that a higher sensitive state of coronary artery to vasoconstrictive agents and a decreased level of vasodilator under thyrotoxic state. 5 In additional, coronary spasm produces a higher chance of atherosclerotic events owing to thrombus formation accelerating and fibrinolysis delaying. Thyrotoxicosis also leads to a hyper metabolic state and causes imbalance between blood supply and oxygen demand, resulting in cardiac symptoms.The abnormalities of the coagulation system have been reported in patients with thyrotoxicosis. Thromboembolism usually occurs in thyrotoxic patients with accompanying atrial fibrillation or congestive heart failure. ${ }^{6}$

Erem et al. reported increased levels of plasma fibrinogen and several coagulation factors, von-Willebrand factor, antithrombin, and PAI-1 and decreased levels of t-PA in patients with overt hyperthyroidism. ${ }^{7}$ Thyrotoxic stage can influence cardiovascular hemodynamic by increasing heart rate, cardiac contractibility, cardiac output, and decreasing systemic vascular resistance. Thyrotoxic stage is a hyper-adrenergic state, where there is increased adrenergic receptor sensitivity and an increased number of receptors, which leads to stimulation of adrenergic receptors on coronary arteries. This may further provoke coronary vasospasm. ${ }^{8}$ Squizzato et al. described increased incidence of acute cerebral ischemia in subjects with hyperthyroidism that was independent of thyrotoxic atrial fibrillation and cardio-embolic stroke. More recently Homoncik et al. ${ }^{7}$ Have reported raised concentrations of vWF and increased baseline platelet plug formation in patients with thyrotoxicosis. In our above patient who had no other condition which could precipitate the acute coronary event at such a young age. There are also reports of severe coronary artery spasm leading to a myocardial infarction in young subjects with thyrotoxicosis; however, without classical cardiovascular disease risk factors. ${ }^{8}$ Furthermore coronary artery spasm seems to be more common in smokers. ${ }^{3}$ While our patient smoked about 15 cigarettes a day. This was the interesting case that was encountered where a young person presented with acute myocardial infarction due to onset of thyrotoxicosis.

CONCLUSION: Our case highlights the possibility of acute myocardial infarction in a young 30 year old male due to thyrotoxicosis with no previous history of thyroid disorder or any vasculitis, thrombosis or other structural or functional heart diseases. Only risk factor present was the smoking which could lead to prothrombotic state combined with thyrotoxicosis and resulted in acute coronary syndrome in a young person. Therefore the possibility of such complications can't be ruled out in young subjects of thyrotoxicosis and needs to be taken care of. 


\section{BIBLIOGRAPHY:}

1. J. Larry Jameson, Anthony P. Weetman/Harrison's internal medicine $18^{\text {th }}$ edition/2012/united states of America/ chapter341/page 2911.

2. Gullu S, Sav H, Kamel N: Effects of levothyroxine treatment on biochemical and hemostasis parameters in patients with hypothyroidism. Eur J Endocrinol 2005, 152:355-361.

3. Andrew Ying-Siu Lee, Michael Chih-Kuang Chang, Tien-Jen Chen (2003) Acute Coronary Syndrome and Hyperthyroidism. Acta Cardiol Sin 19: 201-204.

4. Kohno A, Hara Y (2001) severe myocardial ischemia following hormone replacement in two cases of hypothyroidism with normal coronary arteriogram. Endocr J 48: 565-572.

5. Jung CH, Rhee EJ, Shin HS, et al. Higher serum free thyroxine levels are associated with coronary artery disease. Endocrine J. 2008; 55:819-826. [PubMed]

6. Yun $\mathrm{KH}$, Jeong $\mathrm{MH}$, Oh SK, et al. Relationship of thyroid stimulating hormone with coronary atherosclerosis in angina patients. Int J Cardiol. 2007; 122:56-60. [PubMed]

7. Erem C, Ersoz HO, Karti SS, UkinÃ§ K, Hacihasanoglu A, et al. (2002) Blood coagulation and fibrinolysis in patients with hyperthyroidism. J Endocrinol Invest 25: 345-350.

8. Hammond HK, White FC, Buxton IL, Saltzstein P, Brunton LL, et al. (1987) Increased myocardial beta-receptors and adrenergic responses in hyperthyroid pigs. Am J Physiol 252: H283-90.

\section{AUTHORS:}

1. Surbhi Chaturvedi

2. Niharika Sharma

3. Ankit Manglunia

4. Prakhar Garg

5. Ganesh Narain Saxena

\section{PARTICULARS OF CONTRIBUTORS:}

1. $3^{\text {rd }}$ Year Resident, Department of Medicine, Mahatma Gandhi Medical College \& Hospital, Jaipur.

2. $3^{\text {rd }}$ Year Resident, Department of Medicine, Mahatma Gandhi Medical College \& Hospital, Jaipur.

3. $2^{\text {nd }}$ Year Resident, Department of Medicine, Mahatma Gandhi Medical College \& Hospital, Jaipur.

FINANCIAL OR OTHER COMPETING INTERESTS: None
4. $3^{\text {rd }}$ Year Resident, Department of Medicine, Mahatma Gandhi Medical College \& Hospital, Jaipur.

5. Professor, Department of Medicine, Mahatma Gandhi Medical College \& Hospital, Jaipur.

\section{NAME ADDRESS EMAIL ID OF THE CORRESPONDING AUTHOR:}

Dr. Surbhi Chaturvedi, 83/29 B, C Neelgiri Marg, Mansarovar, Jaipur.

E-mail: ankit.manglunia@yahoo.in

Date of Submission: 08/06/2015. Date of Peer Review: 09/06/2015. Date of Acceptance: 26/06/2015. Date of Publishing: 06/07/2015. 